\title{
Composition Operations of Generalized Entropies Applied to the Study of Numbers
}

\author{
Amelia Carolina Sparavigna ${ }^{1}$
}

${ }^{1}$ Department of Applied Science and Technology, Politecnico di Torino, Torino, Italy

\begin{abstract}
The generalized entropies of C. Tsallis and G. Kaniadakis have composition operations, which can be applied to the study of numbers. Here we will discuss these composition rules and use them to study some famous sequences of numbers (Mersenne, Fermat, Cullen, Woodall and Thabit numbers). We will also consider the sequence of the repunits, which can be seen as a specific case of q-integers.
\end{abstract}

Keywords: Generalized Entropies, Q-Calculus, Abelian Groups, Hyperbolic Functions, Fermat Numbers, Mersenne Numbers, Thabit Numbers, Repunits

\begin{abstract}
Introduction
In some recent works [1-3], we have discussed the generalized entropies of C. Tsallis [4] and G. Kaniadakis [5,6], with the aim of applying them to image processing and image segmentation. What is quite attractive of these entropies is the fact that they are non-additive. It means that they are following rules of composition, which are different from the usual operation of addition. Moreover, these composition rules contain indices, which are useful to have a specific segmentation of images, or even to drive a gray-level image transition among the textures of the image [7].
\end{abstract}

The Tsallis composition rule is defined in [8] as a pseudo-additivity. However, as these rules are concerning generalized entropies, we could call them "generalized sums". Actually, I used this locution in a discussion about the rules of composition that we can obtain from the transcendental functions [9]. The approach, given in [9], is using a method based on the generators of algebras [10-12]. Here I show that we can use the generalized sums, as those that we can obtain from Tsallis and Kaniadakis generalized statistics, to the study of the sequences of numbers. In particular, we will discuss the composition rules that can be applied to famous sequences of numbers, such as Fermat, Mersenne and Thabit numbers. We will also consider the sequence of the repunits, which are a specific case of q-integers.

Let us start remembering the composition rules of Kaniadakis and Tsallis entropies.

The generalized sum of Kaniadakis statistics In [6], a generalized sum is defined in the following manner. Let us consider two elements $x$ and $y$ of reals
$\mathbf{R}$, and a parameter $\kappa$ real too. The composition law $x \oplus y$ is given by:

$x \oplus y=x \sqrt{1+\kappa^{2} y^{2}}+y \sqrt{1+\kappa^{2} x^{2}}$

which defines a generalized sum, named $\kappa$-sum. Reals R and operation (1) form an Abelian group.

Let us remember that a group is a set $A$ and an operation $\bullet$. The operation combines any two elements $a, b$ to form another element of the group denoted $a \bullet b$.

To qualify $(A, \bullet)$ as a group, the set and operation must satisfy the following requirements. Closure: For all $a, b$ in $A$, the result of the operation $a \bullet b$ is also in $A$. Associativity: For all $a, b$ and $c$ in $A$, it holds $(a \bullet b)^{\bullet} c=$ $a \cdot\left(b^{\bullet} c\right)$. Identity element: An element $e$ exists in $A$, such that for all elements $a$ in $A$, it is $e^{\bullet} a=a \bullet e=a$. Inverse element: For each $a$ in $A$, there exists an element $b$ in $A$ such that $a \bullet b=b \bullet a=e$, where $e$ is the identity (the notation is inherited from the multiplicative operation).

If a group is Abelian, a further requirement is the commutativity. For all $a, b$ in $A, a \bullet b=b \bullet a$. Therefore, to qualify a group as an Abelian group, the set and operation must satisfy five requirements, which are known as the Abelian group axioms. A group having a not commutative operation is a "non-Abelian group" or a "non-commutative group". For an Abelian group, one may choose to denote the group operation by + and the identity element by 0 (neutral element) and the inverse element of $a$ as $-a$ (opposite element). In this case, the group is called an additive group.

This article is published under the terms of the Creative Commons Attribution License 4.0 Author(s) retain the copyright of this article. Publication rights with Alkhaer Publications. Published at: http://www.ijsciences.com/pub/issue/2019-04/

DOI: 10.18483/ijSci.2044; Online ISSN: 2305-3925; Print ISSN: 2410-4477 
We can obtain the operation of a group by means of functions. Actually, if a function $G(x)$ exists, which is invertible $G^{-1}(G(x))=x$, we can use it as a generator, to generate an algebra [10]. In [11], $G$ is used to define the group law $\Phi(x, y)$, such as: $\Phi(x, y)=G\left(G^{-1}(x)+G^{-1}(y)\right) . \quad \Phi(x, y)$ is the operation $x \oplus y$. In the case of the $\kappa$-sum, the function $G$ is the hyperbolic sine, so that [9]:

$x \oplus y=\frac{1}{\kappa} \sinh (\operatorname{arsinh}(\kappa x)+\operatorname{arsinh}(\kappa y))$.

This operation is used in Kaniadakis generalized statistics for the sum of relativistic momenta. A property of the hyperbolic sine function is that:

$\operatorname{arsinh}(x)=\ln \left(x+\sqrt{1+x^{2}}\right)$

The domain is the whole real line. We have [13]:

$\operatorname{arsinh}(x) \pm \operatorname{arsinh}(y)$

$=\operatorname{arsinh}\left(x \sqrt{1+y^{2}} \pm y \sqrt{1+x^{2}}\right)$

Therefore, we have that the group law is given as [9]: $\Phi(x, y)=\sinh (\operatorname{arsinh}(x)+\operatorname{arsinh}(y))$.

Therefore, the generalized sum is:

$x \oplus y=x \sqrt{1+y^{2}}+y \sqrt{1+x^{2}}$

This is the same as (1), for parameter $\kappa=0$. The closure is given by the fact that the result of this operation is on the real line. The neutral element is 0 . The opposite element of $x$ is $-x$. Also the commutativity is evident.

To have a group, we need the discussion of the associativity too, showing therefore that $(x \oplus y) \oplus$ $z=x \oplus(y \oplus z)$. Let us calculate $\operatorname{arsinh}((x \oplus$ $y) \oplus z)$ and $\operatorname{arsinh}(x \oplus(y \oplus z))$; we can easily see that [9]: $\operatorname{arsinh}((x \oplus y) \oplus z)=\operatorname{arsinh}(x \oplus$ $(y \oplus z))=\operatorname{arsinh}(x)+\operatorname{arsinh}(y)+\operatorname{arsinh}(z)$.

\section{Other groups}

Of course, other generalized sums can be obtained. Let us consider, for instance, the hyperbolic tangent. Its inverse hyperbolic function is defined as:

$\operatorname{artanh}(x)=\frac{1}{2} \ln \left(\frac{1+x}{1-x}\right)$

The domain is the open interval $(-1,1)$. A property of this function is the following [13]:

$\operatorname{artanh}(x) \pm \operatorname{artanh}(y)=\operatorname{artanh}\left(\frac{x \pm y}{1 \pm x y}\right)$
We have a group law given by $\Phi(x, y)=$ $\tanh (\operatorname{artanh}(x)+\operatorname{artanh}(y))$. Therefore, we can obtain the generalized sum as [9]:

$x \oplus y=(x+y) /(1+x y)$

This sum is commutative. The neutral element is 0 . The opposite element of $x$ is $-x$. We have the associativity, that is $(x \oplus y) \oplus z=x \oplus(y \oplus z)$ [9].

To show that we have a group of $(-1,1)$ and operation (3) it is necessary to verify the closure. That is, we have to see that the result of the sum is in the open interval $(-1,1)$. This is discussed in [9].

The generalized sum (3) is used by Kaniadakis in Ref.14 for the relativistic velocity.

Another group is obtained from function $G(x)=$ $e^{-2 x}\left(e^{2 x}-1\right)$ and its inverse $G^{-1}(x)=\ln \left(\frac{1}{\sqrt{x-1}}\right)$ The group law $\Phi(x, y)=G\left(G^{-1}(x)+G^{-1}(y)\right)$ gives [9]:

$x \oplus y=(1-x)+(1-y)+x y$

We will show in a following section that the same rule of additivity exists for the Fermat numbers.

\section{The q-integers}

Let us see how we can apply the previously discussed generalized sums to the numbers. Let us start from the q-integers of the q-calculus.

Many mathematicians have contributed to this calculus [15-20]. Consequently, the q-calculus is also known as "quantum calculus" and "time-scale calculus", or "calculus of partitions" too [19]. Moreover, it is expressed by means of different notations or, as told in [19], by different "dialects". Here we will use the notation given in the book by Kac and Cheung [20].

As discussed in [21], the q-integers are forming a group having a generalized sum, which is similar to sum of the Tsallis q-entropy of two independent systems. The symmetric q-integers are linked to the Kaniadakis calculus.

The "q-integer" $[n]$ is defines by:

$[n]=\frac{q^{n}-1}{q-1}=1+q+q^{2}+\ldots+q^{n-1}$

First, we have to define the operation of addition by composing two q-integers. This operation is not the sum that we use for the natural integers of course, but it is a generalized sum.

Let us start from the q-integer $[m+n]$ and calculate as in [21]. We have: 
$[m+n]=[m]+[n]+(q-1)[m][n]$

Then, we can define the generalized "sum" of the group as:

$[m] \oplus[n]=[m]+[n]+(q-1)[m][n]$

If we use (5) as the sum, we have the closure of it, because the result of the sum is a q-integer. Moreover, this sum is commutative. The neutral element is $[0]=0$. The opposite element of $[n]$ is equal to $[-n][21]$.

The generalized sum (5) is associative [21]. We have also that: $[m] \oplus[n] \oplus[l]=[m+n+l]$.

Therefore, the five axioms of an Abelian group are satisfied. In this manner, using the generalized sum given by (5), we have the Abelian group of the qintegers.

What is important for the present discussion is the fact that the generalized sum (5) is similar to the sum that we find in the approach to entropy proposed by Constantino Tsallis [4], for his generalized entropy. For two independent systems $A$ and $B$, the Tsallis entropy is given by:

$S_{q}(A, B)=S_{q}(A)+S_{q}(B)+(1-q) S_{q}(A) S_{q}(B)$

In this formula the parameter $(1-q)$, in a certain manner, measures the departure from the ordinary additivity, which is recovered in the limit $q \rightarrow 1$. The group on which is based the Tsallis entropy, and therefore the generalized sum given above, is known in literature as the "multiplicative group". As told in [12], the use of a group structure allows determining a class of generalized entropies.

\section{Symmetric q-numbers}

In the previous section, we have considered the group of the q-integers as defined by q-calculus. In [20], it is also defined the symmetric q-integer in the following form (here we use a notation different from that given in the Ref.20):

$[n]_{s}=\frac{q^{n}-q^{-n}}{q-q^{-1}}$

Repeating the approach previously given, we can determine the group of the symmetric q-integers. Let us start from the q-integer $[m+n]_{s}$, which is:

$[m+n]_{S}=\frac{q^{m+n}-q^{-(m+n)}}{q-q^{-1}}$

and try to find it as a generalized sum of the qintegers $[m]_{S}$ and $[n]_{S}[21]$.
By writing $q=\exp (\ln q)$, the q-integer turns out into a hyperbolic sine:

$$
\begin{aligned}
& {[n]_{S}=\frac{q^{n}-q^{-n}}{q-q^{-1}}=} \\
& \frac{e^{n \ln q}-e^{-n \ln q}}{q-q^{-1}}=2 \frac{\sinh (n \ln q)}{\left(q-q^{-1}\right)}
\end{aligned}
$$

from (6), after some passages using (7), we find [21]:

$$
\begin{aligned}
& {[m+n]_{s}=[m]_{s} \sqrt{1+\sinh ^{2}(n \ln q)}+} \\
& {[n]_{s} \sqrt{1+\sinh ^{2}(m \ln q)}}
\end{aligned}
$$

Let us define: $k=\left(q-q^{-1}\right) / 2$ and then: $k[n]_{s}=$ $\sinh (n \ln q)$. Therefore, we have the generalized sum of the symmetric q-integers as:

$$
\begin{aligned}
& {[m]_{s} \oplus[n]_{s}=} \\
& \quad[m]_{s} \sqrt{1+k^{2}[n]_{S}^{2}}+[n]_{s} \sqrt{1+k^{2}[m]_{S}^{2}}
\end{aligned}
$$

Let us stress that (8) is also the generalized sum (1) proposed by G. Kaniadakis (see also the discussion in [22]).

\section{The Mersenne numbers}

In the case that $q=2$, we have:

$$
[n]=\frac{2^{n}-1}{2-1}=2^{n}-1 .
$$

These are the Mersenne Numbers.

About these numbers, a large literature exists (see for instance that given in [23]). Among the Mersenne integers, we find the Mersenne primes. The numbers are named after Marin Mersenne (1588 - 1648), a French Minim friar, who studied them in the early 17th century. Let us call $M_{n}$ the Mersenne number. Of course, we have the generalized sum for the qnumbers as given in (5):

$$
[m] \oplus[n]=[m]+[n]+[m][n]
$$

However, we can repeat the calculus starting from $M_{m+n}=2^{m+n}-1$. After some passages we obtain $M_{m+n}=\left(2^{m}-1\right)\left(2^{n}-1\right)+2^{m}-1+2^{n}-1$, that is:

$M_{m+n}=M_{m} \oplus M_{n}=M_{m}+M_{n}+M_{m} M_{n}$

Let us stress that we have a generalized sum of the form of those of the groups known as "multiplicative groups".

Using (9), for the Mersenne numbers we can imagine the following recursive relation:

$$
M_{n+1}=M_{n} \oplus M_{1}=M_{n}+M_{1}+M_{n} M_{1}
$$


The sum (9) is associative.

We cannot have a group of the Mersenne numbers, without considering also the opposites $O$ of them, so that: $0=M_{n} \oplus O\left(M_{n}\right)$. Therefore:

$O\left(M_{n}\right)=\frac{-M_{n}}{M_{n}+1}=M_{-n}$

Explicitly: $O\left(2^{n}-1\right)=2^{-n}-1$. These numbers are the Mersenne numbers with a negative exponent.

\section{Symmetric Mersenne}

Let us consider again the symmetric q-integer in the case of $q=2$.

We can define the symmetric Mersenne in the following manner:

$M_{n}^{s}=[n]_{s}=\frac{2^{n}-2^{-n}}{2-2^{-1}}$

By writing $2=\exp (\ln 2), \quad(10)$ turns out into a hyperbolic sine:

$M_{n}^{s}=\frac{e^{n \ln 2}-e^{-n \ln 2}}{2-2^{-1}}=2 \frac{\sinh (n \ln 2)}{\left(2-2^{-1}\right)}$

Let us define $k=\left(2-2^{-1}\right) / 2$. We have the generalized sum of the symmetric Mersenne as:

$M_{m}^{s} \oplus M_{n}^{s}=$
$M_{m}^{s} \sqrt{1+k^{2}\left(M_{n}^{s}\right)^{2}}+M_{n}^{s} \sqrt{1+k^{2}\left(M_{m}^{s}\right)^{2}}$

Of course, we have again the generalized sum proposed by G. Kaniadakis.

\section{Fermat, Cullen and Woodall Numbers}

As seen before, the Mersenne numbers $M_{n}=2^{n}-1$ are forming a group with the following generalized sum:

$M_{m} \oplus M_{n}=M_{m}+M_{n}+M_{m} M_{n}$

Using this composition rule, we can have the following recursive relation:

$M_{n+1}=M_{n} \oplus M_{1}=$

$M_{n}+M_{1}+M_{n} M_{1}=2 M_{n}+1$

Numbers are $1,3,7,15,31,63,127,255,511,1023$, 2047, 4095, 8191, 16383, 32767, 65535, and so on, in agreement to the sequence given in http://oeis.org/A000225.

Another famous sequence of integers is that of the Fermat numbers. Fermat numbers are defined as $F_{n}=2^{n}+1$ [24]. These numbers have the following generalized sum [25]:
$F_{m} \oplus F_{n}=\left(1-F_{m}\right)+\left(1-F_{n}\right)+F_{m} F_{n}$

which is the same of the group law (4) that we have discussed in [25] and in a previous section of this work.

From (12), we can give the following recursive relation for the Fermat numbers: $F_{n+1}=F_{n} \oplus F_{1}$.

Starting from $F_{1}=2^{1}+1=3$, we have: $3,5,9,17$, $33,65,129,257,513,1025,2049,4097,8193$, $16385,32769,65537,131073,262145,524289$, and so on in agreement to http://oeis.org/A000051.

The sum is associative. The neutral element is $F_{0}=2^{0}+1=2$ and the opposites $O$ of the Fermat numbers are given by $O\left(F_{n}\right)=F_{-n}[25]$.

Similar to the Fermat numbers, we have the Cullen numbers. The Woodall numbers are similar to the Mersenne numbers [26,27]. Let us find the generalized sums of them, as detailed in [28].

The Cullen numbers are $C_{n}=2^{n} n+1$.

The generalized sum is [28]:

$C_{m} \oplus C_{n}=2^{m}\left(C_{n}-1\right)+2^{n}\left(C_{m}-1\right)+1$

The neutral element of this sum is $C_{0}=2^{0} 0+1=1$. Using (13):

$C_{m} \oplus C_{0}=$

$2^{m}\left(C_{0}-1\right)+2^{0}\left(C_{m}-1\right)+1=C_{m}$

We have $C_{1}=2^{1} 1+1=3$. Recursive relation is:

$C_{n+1}=2^{n+1}+2\left(C_{n}-1\right)+1$

So we have: $3,9,25,65,161,385,897,2049,4609$, 10241, 22529, 49153, 106497, 229377, 491521, 1048577, and so on, in agreement to http://oeis.org/A002064.

Of the Cullen numbers, we can also give another form of the generalized sum [28]:

$C_{m} \oplus C_{n}=$

$\frac{1}{m}\left(C_{m}-1\right)\left(C_{n}-1\right)+\frac{1}{n}\left(C_{n}-1\right)\left(C_{m}-1\right)+1$

The recursive relation assumes the form: $C_{n+1}=$ $2\left(C_{n}-1\right)+\frac{2}{n}\left(C_{n}-1\right)+1$. In the case that we use the generalized sum (14), we have to remember that when $m$ or $n$ are equal to zero, we need to assume $\left(C_{m}-1\right) / m=1,\left(C_{n}-1\right) / n=1$.

The Woodall numbers are defined as: $W_{n}=2^{n} n-1$. Let us write the generalized sum as [28]:

$W_{m} \oplus W_{n}=2^{m}\left(W_{n}+1\right)+2^{n}\left(W_{m}+1\right)-1$ 
The neutral element of this sum is $W_{0}=2^{0} 0-1=$ -1 . Using (15):

$W_{m} \oplus W_{0}=$

$2^{m}\left(W_{0}+1\right)+2^{0}\left(W_{m}+1\right)-1=W_{m}$

We have $W_{1}=2^{1} 1-1=1$. Recursive relation is:

$W_{n+1}=2^{n+1}+2\left(W_{n}+1\right)-1$

So we have: $1,7,23,63,159,383,895,2047,4607$, 10239, 22527, 49151, 106495, 229375, 491519, 1048575, and so on, in agreement to http://oeis.org/A003261.

Also for the Woodall numbers, we can give another form of the generalized sum (15), as shown in [28].

\section{Thabit numbers}

Let us consider the Thabit numbers [29]. These numbers are given as $T_{n}=3 * 2^{n}-1$, where the asterisk represents the ordinary multiplication. The operation of addition between Thabit numbers is [30]:

$T_{m} \oplus T_{n}=\left(T_{m}+T_{n}+T_{m} T_{n}-2\right) / 3$

Using (16), we can see that the neutral element is $T_{0}=2$, so that:

$T_{m} \oplus T_{0}=\frac{1}{3}\left(T_{m}+T_{0}+T_{m} T_{0}-2\right)=T_{m}$

The recursive relation is given accordingly to (16), starting from $T_{1}=5$ :

$T_{n+1}=T_{n} \oplus T_{1}=2 T_{n}+1$

We have 5, 11, 23, 47, 95, 191, 383, 767, 1535, $3071, \mathbf{6 1 4 3}, 12287,24575,49151$, and so on. In bold characters, the prime numbers as from http://oeis.org/A007505.

\section{Repunits}

As explained in [31], the term "repunit" was coined by Beiler in a book of 1966 [32], for the numbers defined as:

$R_{n}=\frac{10^{n}-1}{10-1}$

The sequence of repunits starts with $1,11,111,1111$, $11111,111111, \ldots$ (sequence A002275 in the OEIS, https://oeis.org/A002275). As we can easily see, these numbers are linked to q-integers and Mersenne numbers [33]. The repunits are the q-integers for $\mathrm{q}=10$ : $[n]_{q=10}=\frac{10^{n}-1}{10-1}$

We can use the same approach for the repunits of that given for the q-numbers. Let us consider the following operation (generalized sum): $R_{m+n}=$ $R_{m} \oplus R_{n}$. It is defined in the following manner:

$R_{m} \oplus R_{n}=R_{m}+R_{n}+(10-1) R_{m} R_{n}$

The recursive relation for the repunits, given according to (17) and starting from $R_{1}=1$, is:

$R_{m} \oplus R_{1}=R_{m}+R_{1}+(10-1) R_{m} R_{1}$

That is: $11,111,1111,11111,111111,1111111$,

11111111 , and so on.

As we have considered the symmetric q-integers, we can define the "symmetric" repunits as [33]:

$R_{n, s}=\frac{10^{n}-10^{-n}}{10-10^{-1}}=2 \frac{\sinh (n \ln 10)}{10-10^{-1}}$

The sequence is: $1,10.1,101.01,1010.101$, 10101.0101, 101010.10101, etc.

In this case, the addition is the same as that for the symmetric q-numbers:

$$
\begin{aligned}
& R_{m, s} \oplus R_{n, s}= \\
& R_{m, s} \sqrt{1+k^{2}\left(R_{n, s}\right)^{2}}+R_{n, s} \sqrt{1+k^{2}\left(R_{m, s}\right)^{2}}
\end{aligned}
$$

Here $k=\frac{1}{2}\left(10-\frac{1}{10}\right)$. Let us note that $R_{1, s}=1$.

The recursive formula for the symmetric repunits is:

$$
\begin{aligned}
& R_{n+1, s}=R_{n, s} \oplus R_{1, s}= \\
& R_{n, s} \sqrt{1+k^{2}}+\sqrt{1+k^{2}\left(R_{n, s}\right)^{2}}
\end{aligned}
$$

\section{Conclusion}

In this work, we have discussed the composition operations of generalized entropies (Tsallis and Kaniadakis). These operations can be obtained from some group laws based on functions and their inverses. The group laws can be defined as "generalized sums" because the generalized entropies are motivating them.

The approach using the group operations can be applied to the study of numbers. We have discussed the composition rules for some famous sequences of numbers (Mersenne, Fermat, Cullen, Woodall and Thabit numbers). We have also considered the sequence of the repunits, which can be seen as a specific case of the q-integers. 


\section{References}

1. Sparavigna, A. C. (2015). Shannon, Tsallis and Kaniadakis entropies in bi-level image thresholding. International Journal of Sciences 4(2), 35-43. DOI: 10.18483/ijSci.626

2. Sparavigna, A. C. (2015). Relations between Tsallis and Kaniadakis entropic measures and rigorous discussion of conditional Kaniadakis entropy. International Journal of Sciences, 4(10), 47-50. DOI: 10.18483/ijsci.866

3. Sparavigna, A. C. (2015), On The Generalized Additivity Of Kaniadakis Entropy, International Journal of Sciences 4(2), 44-48. DOI: $10.18483 / \mathrm{ijSci} .627$

4. Tsallis, C. (1988). Possible generalization of BoltzmannGibbs statistics. Journal of Statistical Physics, 52(1-2), 479487. DOI: $10.1007 / \mathrm{bf01016429}$

5. Kaniadakis, G. (2001). Non-linear kinetics underlying generalized statistics. Physica A, 296, 405-425. DOI 10.1016/s0378-4371(01)00184-4

6. Kaniadakis, G. (2013). Theoretical Foundations and Mathematical Formalism of the Power-Law Tailed Statistical Distributions. Entropy, 15, 3983-4010. DOI 10.3390/e15103983

7. Sparavigna, A. C. (2015). Gray-level image transitions driven by Tsallis entropic index. International Journal of Sciences 4(2), 16-25. DOI: 10.18483/ijSci.621

8. Portes De Albuquerque, M., Esquef, I. A., Gesualdi Mello, A R., \& Portes De Albuquerque, M. (2004). Image thresholding using Tsallis entropy. Pattern Recognition Letters, 25(9), 1059-1065. DOI: 10.1016/j.patrec.2004.03.003

9. Sparavigna, A. C. (2018). Generalized Sums Based on Transcendental Functions. SSRN Electronic Journal. Elsevier. DOI: $10.2139 / \mathrm{ssrn} .3171628$

10. Scarfone, A. M. (2013). Entropic forms and related algebras. Entropy, 15(2), 624-649. DOI: 10.3390/e15020624

11. Sicuro, G., \& Tempesta, P. (2016). Groups, information theory, and Einstein's likelihood principle. Phys. Rev. E 93 040101(R). DOI: 10.1103/physreve.93.040101

12. Tempesta, P. (2015). Groups, generalized entropies and Lseries. Templeton Workshop on Foundations of Complexity, October 2015 http://www.cbpf.br/ complex/Files/talk_tempesta.pdf

13. Abramowitz, M., \& Stegun, I. (1964). Handbook of Mathematical Functions with Formulas, Graphs, and Mathematical Tables.

14. Kaniadakis, G. (2005). Statistical mechanics in the context of special relativity II. Phys. Rev. E, 72, 036108. DOI 10.1103/physreve.72.036108

15. Ernst, T. (2012). A Comprehensive Treatment of q-Calculus, Springer Science \& Business Media.
16. Annaby, M. H., \& Mansour, Z. S. (2012). q-Fractional Calculus and Equations, Springer.

17. Ernst, T. (2000). The History of q-calculus and a New Method. Department of Mathematics, Uppsala University.

18. Aral, A., Gupta, V., \& Agarwal, R. P. (2013). Applications of q-Calculus in Operator Theory. Springer Science \& Business Media.

19. Ernst, T. (2008). The different tongues of q-calculus. Proceedings of the Estonian Academy of Sciences, 2008, 57, 2, 81-99 DOI: 10.3176/proc.2008.2.03

20. Kac, V., \& Pokman Cheung (2002). Quantum Calculus, Springer, Berlin.

21. Sparavigna, A. C. (2018). The q-integers and the Mersenne numbers. SSRN Electronic Journal. Elsevier. DOI: $10.2139 / \mathrm{ssrn} .3183800$

22. Sparavigna, A. C. (2018). On the generalized sum of the symmetric q-integers. Zenodo. DOI 10.5281/zenodo.1248959

23. Weisstein, Eric W. "Mersenne Number". From MathWorld A Wolfram Web Resource. http://mathworld.wolfram.com/MersenneNumber.html

24. Weisstein, Eric W. "Fermat Number". From MathWorld - A Wolfram Web Resource. http://mathworld.wolfram.com/FermatNumber.html

25. Sparavigna, A. C. (2018). The group of the Fermat Numbers. Zenodo. DOI: 10.5281/zenodo.1252422

26. Weisstein, Eric W. "Cullen Number". From MathWorld - A Wolfram Web Resource. http://mathworld.wolfram.com/CullenNumber.html

27. Weisstein, Eric W. "Woodall Number". From MathWorld - A Wolfram Web Resource. http://mathworld.wolfram.com/WoodallNumber.html

28. Sparavigna, A. C. (2019). On the generalized sums of Mersenne, Fermat, Cullen and Woodall Numbers. Zenodo. DOI: $10.5281 /$ zenodo.2634312

29. Weisstein, Eric W. (2019). "Thâbit ibn Kurrah Number". From MathWorld - A Wolfram Web Resource. http://mathworld.wolfram.com/ThabitibnKurrahNumber.html

30. Sparavigna, A. C. (2019). A recursive formula for Thabit numbers. Zenodo. DOI: 10.5281/zenodo.2638790

31. Weisstein, Eric W. (2019). "Repunit". From MathWorld - A Wolfram Web Resource. http://mathworld.wolfram.com/Repunit.html

32. Beiler, A. H. (1966). "11111...111." Ch. 11 in Recreations in the Theory of Numbers: The Queen of Mathematics Entertains. New York: Dover.

33. Sparavigna, A. C. (2019). On Repunits. DOI 10.5281/zenodo.2639620 